\title{
High-energy proton induced damage study of scintillation light output from $\mathrm{PbWO}_{4}$ calorimeter crystals
}

\author{
P. Lecomte, D. Luckey, F. Nessi-Tedaldi, F. Pauss \\ Institute for Particle Physics, ETH Zurich, 8093 Zürich, Switzerland
}

\begin{abstract}
Eight $\mathrm{PbWO}_{4}$ crystals produced for the electromagnetic calorimeter of the CMS experiment at LHC have been irradiated in a $20 \mathrm{GeV} / \mathrm{c}$ proton beam up to fluences of $5.4 \times 10^{13} \mathrm{p} / \mathrm{cm}^{2}$. The damage recovery in these crystals, stored in the dark at room temperature, has been followed for over a year. Comparative irradiations with ${ }^{60} \mathrm{Co}$ photons have been performed on seven other crystals using a dose rate of $1 \mathrm{kGy} / \mathrm{h}$. The issue whether hadrons cause a specific damage to the scintillation mechanism has been studied through light output measurements on the irradiated crystals using cosmic rays. The correlation between light output changes and light transmission changes is measured to be the same for proton-irradiated crystals and for $\gamma$-irradiated crystals. Thus, within the precision of the measurements and for the explored range of proton fluences, no additional, hadron-specific damage to the scintillation mechanism is observed.
\end{abstract}




\section{Introduction}

The way large hadron fluxes affect $\mathrm{PbWO}_{4}$ crystals has become relevant with the construction of LHC [1]. For the CMS experiment, charged hadron fluences have been calculated [2] for an integrated luminosity of $5 \times 10^{5} \mathrm{pb}^{-1}$, which nominally corresponds to 10 years of running at LHC, yielding in the electromagnetic calorimeter (ECAL) barrel up to $\sim 10^{12}$ and in the end caps $\sim 10^{14}$ charged hadrons $/ \mathrm{cm}^{2}$. It had to be ascertained whether such fluxes cause a specific, possibly cumulative, damage, and if so, what its quantitative importance is and whether it only affects light transmission (LT) or also the scintillation mechanism. A hadron-specific damage could arise from the production, above a $\sim 20 \mathrm{MeV}$ threshold, of heavy fragments with up to $10 \mu \mathrm{m}$ range and energies up to $\sim 100$ $\mathrm{MeV}$, causing a displacement of lattice atoms and energy losses, along their path, up to 50000 times the one of minimum-ionising particles. The damage caused by these processes is likely to be different from the one caused by ionising radiation and could be cumulative.

In a study we have published recently [3], the primarily investigated quantity was the damage to light transmission, because it can be measured very accurately. Furthermore, all damage observed in earlier tests [4] 5] could always be ascribed to the ionising dose associated with the hadron flux, apart from some indication for a hadron-specific damage in BGO, which can be extracted [6] from existing data. None of the previous tests on lead tungstate was extended however to the full integrated fluences expected at the LHC.

Eight CMS production crystals of consistent quality were irradiated at the IRRAD1 facility of the CERN PS accelerator T7 beam line [7] in a $20 \mathrm{GeV} / \mathrm{c}$ proton flux of either $10^{12} \mathrm{p} \mathrm{cm}^{-2} \mathrm{~h}^{-1}$ (crystals $a, b, c, d, h$ ) or of $10^{13} \mathrm{p} \mathrm{cm}^{-2} \mathrm{~h}^{-1}$ (crystals $\left.E, F, G\right)^{1}$ ). These crystals have the shape of truncated pyramids with nearly parallelepipedic dimensions of $2.4 \times 2.4 \mathrm{~cm}^{2}$ and a length of $23 \mathrm{~cm}$. The exact fluxes and fluences each crystal was exposed to are listed in Ref. [3]. The maximum fluence, of $5.4 \times 10^{13} \mathrm{p} / \mathrm{cm}^{2}$, was reached for crystal $a$ ”. To disentangle the contribution to damage from the associated ionising dose, complementary ${ }^{60} \mathrm{Co} \gamma$-irradiations were performed at a dose rate of $1 \mathrm{kGy} / \mathrm{h}$ on further seven crystals $(t, u, v, w, x, y, z)$ at the ENEA Casaccia Calliope irradiation facility [8], since a flux of $10^{12} \mathrm{p} \mathrm{cm}^{-2} \mathrm{~h}^{-1}$ has an associated ionising dose rate in $\mathrm{PbWO}_{4}$ of $1 \mathrm{kGy} / \mathrm{h}$. The maximum dose of $50.3 \mathrm{kGy}$ was reached for crystal $v$, just a factor $\sim 2$ below the dose reached in proton irradiations, which for $a$ " was $97.5 \mathrm{kGy}$.

The study of transmission damage revealed that proton irradiation decreases the light transmission for all wavelengths and moves the ultra-violet LT band-edge to longer wavelengths, as can be seen in Fig. 9 of Ref. [3]. In $\gamma$-irradiated crystals, the band-edge does not shift at all, even after the highest cumulated dose: only the well-known absorption band [9] appears around $420 \mathrm{~nm}$. These data demonstrate the qualitatively different nature of protoninduced and $\gamma$-induced damage. The correlation we found, between the induced absorption coefficient at the peak of scintillation-emission wavelength, $\mu_{I N D}(420 \mathrm{~nm})$ [3], and fluence, is consistent with a linear behaviour over two orders of magnitude, showing that proton-induced damage in $\mathrm{PbWO}_{4}$ is predominantly cumulative, unlike $\gamma$-induced damage, which reaches equilibrium [9, 10]. The data for $\mu_{I N D}$ versus light wavelength for protonirradiated crystals show a $\lambda^{-4}$ dependence, which is absent in $\gamma$ - irradiated crystals. This is an indication of Rayleigh scattering from small centres of severe damage, as it might be caused by the high energy deposition of heavily ionising fragments along their path, locally changing the crystal structure. Taking into account the difference in composition and energy spectra between $20 \mathrm{GeV} / \mathrm{c}$ protons and CMS, simulations indicate that the test results cover the CMS running conditions up to a pseudorapidity of $\sim 2.6$. An experimental confirmation is expected from a planned pion-irradiation of $\mathrm{PbWO}_{4}$, closer to the CMS particle spectrum average [3].

However, the relevant quantity for detector operation is the scintillation light output (LO). Detector calibration through a light injection system, as foreseen by CMS [11], can only work, as long as changes in light output are all due to changes in light transmission. This had been verified extensively so far for ionising radiation, but for high hadron fluxes it still had to be established: the present work deals with this question. In the following, the measurement setup and technique are described, the data analysis is explained, and results are given, in comparison, for proton-irradiated crystals and for $\gamma$-irradiated crystals.

\section{The setup}

Light output from a crystal is commonly measured in the laboratory using photons from a ${ }^{60} \mathrm{Co}$ or ${ }^{137} \mathrm{Cs}$ source to excite scintillation. The light output is then determined from the position, in the spectrum, of the photoelectric peak produced by photons which deposit all their energy in the crystal. For lead tungstate crystals, the light output

1) Labels 'prime' (respectively 'double-prime') will be used after the letter identifying the crystal, in order to indicate a second (or third) irradiation of the same crystal. 
is modest, $\sim 10$ photoelectrons/MeV of incoming particle energy, as detected on the bialkali photocathode of a photomultiplier (PM) covering the whole back face of the crystal. Thus, the photoelectric peak is broadened by statistical fluctuations and merged with the Compton edge. In the present study, the hadron-irradiated crystals will remain radioactive for years, and thus act as sources for $\gamma$ and $\beta$ emission over an extended range of energies, peaked at the lowest detectable values and tailing off towards larger ones. The photoelectric peak from any common laboratory $\gamma$ source is swamped by this spectrum, and a different method of LO determination had to be adopted.

As an alternative, cosmic muons passing a crystal transversely were used. These muons deposit $24.5 \mathrm{MeV}$ in the crystal, an energy deposit which is well above all $\gamma$ energies emitted by radioactive isotopes. Using these muons to excite scintillation gave us a light output well above the crystal's own background.

An individual crystal was placed in horizontal position, with plastic scintillator counters above and below, to select nearly vertical muons. Since only a relative measurement of LO loss was sought, large rectangular plastic scintillators were used, $24 \mathrm{~cm}$ long and $23 \mathrm{~cm}$ wide, sitting at $22 \mathrm{~cm}$ distance above and below the crystal. These counters yielded a large angular acceptance, allowing for muons traversing the crystal at angles between $0^{\circ}$ and $37^{\circ}$ from the vertical, leaving thus an energy deposit $E_{\text {dep }}$ between $24.5 \mathrm{MeV}$ and $30.6 \mathrm{MeV}$.

\section{Measurements and systematics}

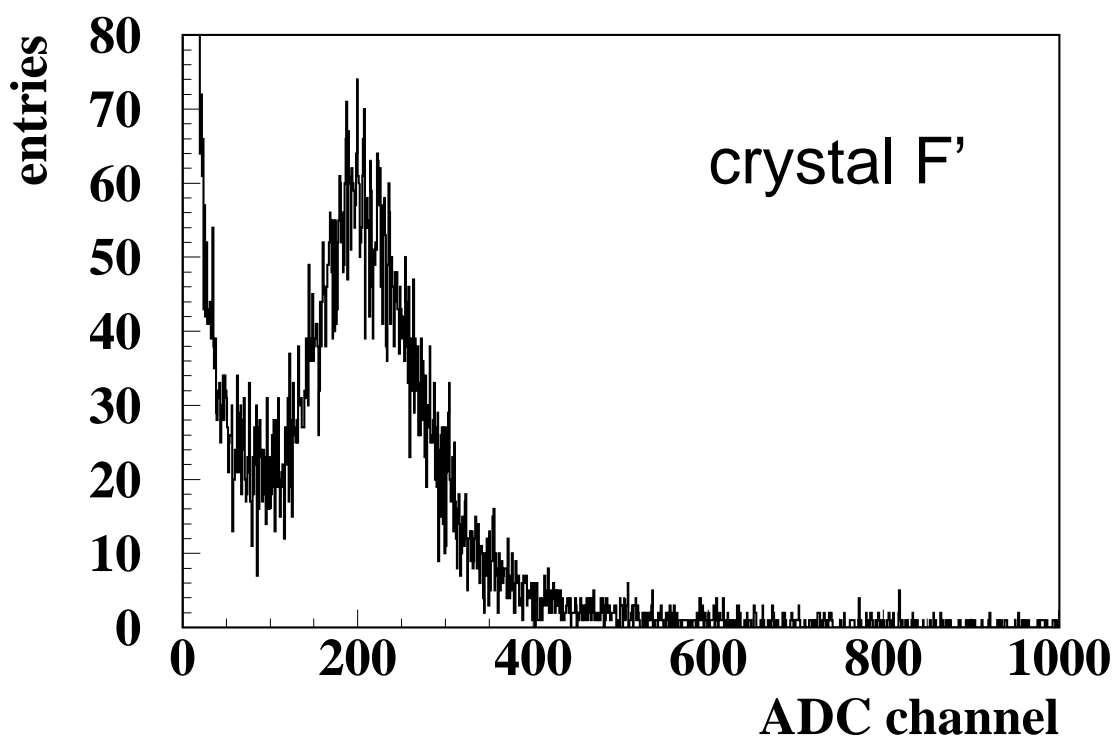

Figure 1: Spectrum for crystal $F^{\prime}$.

For LO measurements, each crystal was wrapped in Tyvek ${ }^{\mathrm{TM}}$ [12] on all sides but its large end, which was coupled via an air gap to a Philips XP2262B bialkali-photocathode PM equipped with a standard CERN base type 4238 . A PVC centering tube was used for reproducible positioning. The photomultiplier was operated at a gain of $\sim 10^{8}$. The current pulses from its anode were fed into an Analog-to-Digital converter 2249W from LeCroy, which has a sensitivity of $0.25 \mathrm{pC}$. Data acquisition was performed with a PC running a Labview 7.0 virtual instrument, interfaced via a HYTEC 1331 controller [13] to a CAMAC crate. The data acquisition was triggered by a coincidence of the signals in both plastic scintillator counters. A CAMAC-controlled Programmable Dual Gate and Delay Generator LeCroy 2323A defined a $200 \mathrm{~ns}$ ADC charge integration time. The acquisition of a spectrum with sufficient statistics for one LO measurement typically took $24 \mathrm{~h}$, yielding approximately 8000 events in the muon energy deposition peak. The ambient temperature was recorded at the beginning and at the end of each measurement, since the lead tungstate $\mathrm{LO}$ changes by $-2 \% /{ }^{\circ} \mathrm{C}[14][1]$ at room temperature, and the light output determined for each run was corrected offline to its value at $18^{\circ} \mathrm{C}$. The uncertainty on the correction, coming from possible, undetected fluctuations of temperature in the crystal over the duration of a run, amounts to $0.4 \%$ and is negligible compared to the other error sources affecting the LO determination, as discussed below. The anode signal was attenuated at the input, by typically $24 \mathrm{db}$, for the acquisition of a muon spectrum. Keeping the same PM high voltage, the stability of the setup, from the PM multiplication chain to the ADC, was monitored by 
periodically acquiring the spectrum of single photoelectrons thermally emitted by the photocathode. In this case, the anode signal from the PM coupled to the crystal was used for self-triggering, with no attenuation before the ADC input, and the discriminator threshold was set low enough to see the valley below the single photoelectron peak, according to the method we described in Ref. [10]. Pedestal data were acquired at the beginning of every run, and subtracted on a run-by-run basis. Since we could not exclude a priori that the crystal radioactivity would affect the detection efficiency of the setup over time, we also periodically monitored the stability of the setup by acquiring the cosmic muons spectrum from a non-irradiated reference crystal, and, when necessary, corrected all data for relative gain changes. This procedure allowed to monitor the whole light detection chain, including the photocathode quantum efficiency.

As an example, Figure 1 shows a cosmic muons spectrum for $F^{\prime}$ after an irradiation with $9.83 \times 10^{12} \mathrm{p} / \mathrm{cm}^{2}$, where the crystal experienced a considerable damage in light transmission, corresponding to an induced absorption coefficient of $\sim 3 \mathrm{~m}^{-1}$. The sharp rise which is clearly visible below channel 100 is due to light produced by the radioactivity of the crystal.

\section{Results}

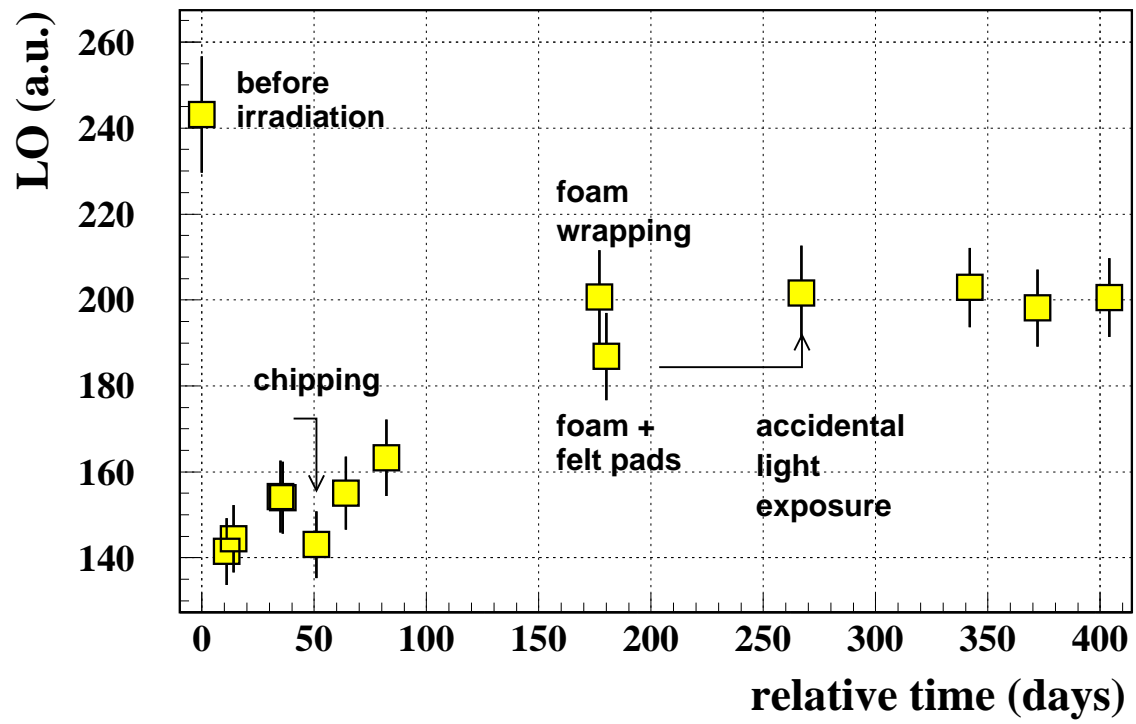

Figure 2: Light output change over time for crystal $G$, where the long-term, natural room-temperature recovery of damage can be observed, along with some accidental changes (see text).

Great care had to be given to sources of systematic errors in the measurements. For precise relative LO loss measurements, the geometrical acceptance of each crystal for triggered muons was kept constant from one measurement to the next, to ensure keeping the average muon energy deposit, $\left\langle E_{d e p}\right\rangle$, always at the same value. This was achieved by placing each crystal every time in the same position and orientation in the setup. The remaining uncertainty has been evaluated from the fluctuation of the various LO measurements for the reference crystal. This was done separately for the period where proton-irradiated crystals were measured, whose irradiations were performed between May and October 2003, and for the period where $\gamma$-irradiated crystals were monitored, which were exposed to protons in May 2004 and measured over the following 7 months. Reproducibility of positioning was significantly improved over time as a result of the learning process. The corresponding, relative uncertainty on LO measurements was determined as the $\sigma$ of the distribution of values for the reference crystal. For $\gamma$-irradiated crystals it amounts to $1.7 \%$ and for proton-irradiated ones, to is $2.4 \%$, except on the data before irradiation for crystals $a$ and $F$, which were taken before monitoring the system stability with frequent reference crystal measurements, and where it amounts to 3.8\%. This uncertainty affects every LO measurement, and it is thus added in quadrature to the statistical errors. However, the LO change, which we want to correlate with the damage to light transmission, is determined as the ratio between light output at a given time after irradiation and the light output before irradiation. The uncertainty on the light output measurement before irradiation thus affects, as an overall scale uncertainty, the whole set of data for each crystal. 

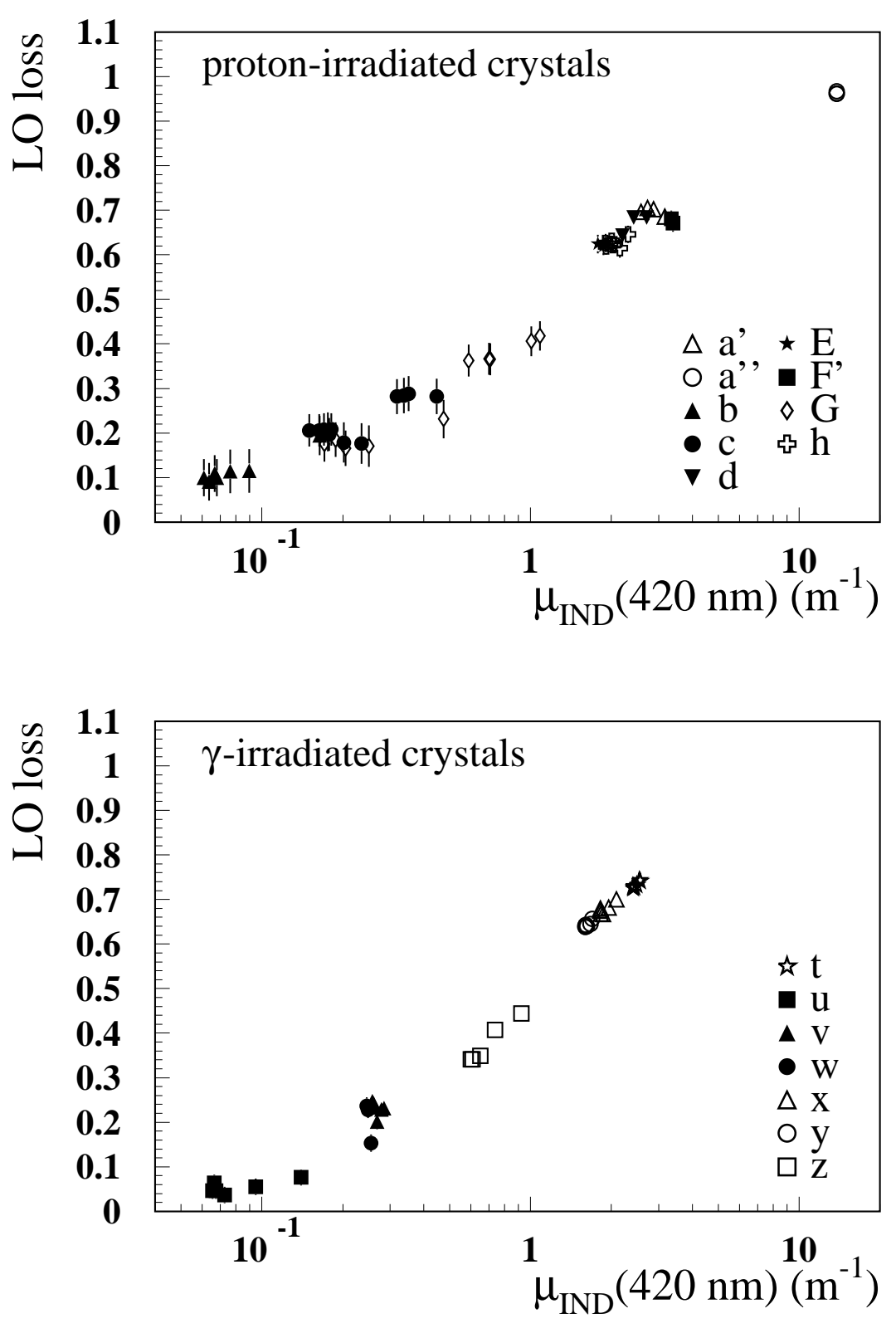

Figure 3: Correlation between $\mu_{I N D}(420 \mathrm{~nm})$ and LO loss for proton-induced (top) and $\gamma$-induced (bottom) damage. See the text for the systematic scale uncertainty on the data (not shown).

Direct contact was initially used between crystal and PM face, but it resulted, for the reference crystal and for the proton-irradiated ones, in the chipping of a few corners, due to the repeated positioning of the crystals in the setup. This caused a small, but noticeable loss of collected light with respect to the natural, room-temperature damage recovery (see Fig. 2), since the surface of the large face was slightly reduced by the accident. To reduce the risk of chipping, a sheet of polyethylene foam was thus first wrapped around the Tyvek, to protect the long chamfers. This resulted in a small rise of LO signal (see Fig. 2) because of the slight change of crystal positioning on the photocathode, whose efficiency is position-dependent. Further, four tiny felt pads were applied, as a protection, to the corners of the big end face, masking $6 \%$ of it. The LO decreased slightly this way, due to the reduced area for light collection (Fig. 2]. Since the amount of chipping was different from crystal to crystal, and since the changes cannot be necessarily disentangled from damage recovery, no correction was applied to the data for these systematic shifts, but we include a relative systematic uncertainty of $4 \%$ from this effect on the LO measurements for pirradiated crystals. The crystals irradiated with photons are not affected by this uncertainty, since the protection was adopted before the reference data taking prior to irradiation. Furthermore, for crystal G one should notice in 
Fig. 2 2 the LO difference between the value at 180 days and the one at 270 days after irradiation: this rise is due to a step in the damage recovery, as it can be appreciated in the corresponding LT recovery data of Fig.11 in Ref. [3], which originated from an accidental, prolonged exposure of the crystal to fluorescent room light.

The correlation between $\mu_{I N D}(420 \mathrm{~nm})$ and LO loss is shown at the top of Fig. 3 for all proton-irradiated crystals. One should notice the very important damage for $a$ ”, which reached $\mu_{I N D}(420 \mathrm{~nm}) \simeq 15 \mathrm{~m}^{-1}$ after a fluence of $5.4 \times 10^{13} \mathrm{p} / \mathrm{cm}^{2}$ and experienced a corresponding LO loss of nearly $96 \%$. The correlation for all $\gamma$-irradiated crystals is shown in the bottom part of Fig. 3. The overall, relative, systematic scale uncertainty affecting the results amounts to $1.7 \%$ for $\gamma$-irradiated crystals and to $4.7 \%$ for p-irradiated ones, except for crystals $a$ and $F$, where it is $5.5 \%$.

Within the precision of our measurements, a clear correlation is observed between loss of light transmission, expressed through $\mu_{I N D}(420 \mathrm{~nm})$, and the relative LO loss, both, for hadron-irradiated crystals and for $\gamma$ irradiated ones. Furthermore, the two correlations are compatible within the precision of our measurements. Thus, in the range of proton fluxes and fluences used for the present irradiations, no hadron-specific alteration to the crystal scintillation properties has been observed.

\section{Conclusions}

A set of lead tungstate crystals of CMS production quality have been exposed to $20 \mathrm{GeV} / \mathrm{c}$ proton fluxes up to $5.4 \times 10^{13} \mathrm{p} / \mathrm{cm}^{2}$. For comparison, a different set of similar quality lead tungstate crystals have been exposed to an equivalent amount of ${ }^{60} \mathrm{Co} \gamma$-radiation in terms of ionising dose rate and dose. Measurements of changes in scintillation light output have been performed and correlated with light transmission changes. Within the precision of our measurements, the correlations for proton-irradiated and for $\gamma$-irradiated crystals are compatible. Thus, for the crystals tested, and within the range of particle fluxes and fluences considered, we do not observe any additional, hadron-specific alteration of lead tungstate scintillation properties.

\section{Acknowledgments}

We are indebted to R. Steerenberg, who provided us with the required PS beam conditions for the proton irradiations. We are deeply grateful to M. Glaser and F. Ravotti, who helped us in operating the irradiation and dosimetry facilities. The support and practical help of S. Baccaro and A. Cecilia during the $\gamma$-irradiations at ENEA-Casaccia were essential and are gratefully acknowledged, as is the contribution of E. Auffray who provided us with the crystals after having characterised their $\gamma$-radiation hardness at the Geneva Hospital. Mika Huhtinen contributed in an invaluable way [3] to the crystal irradiations and to the work on LT damage.

\section{References}

[1] F. Nessi-Tedaldi, Int. J. Mod. Phys. A20 (2005) 3826-3829.

[2] The CMS Collaboration, The Tracker Project Technical Design Report, CERN/LHCC 98-6, CMS TDR 5, CERN, Geneva (Switzerland, 1998).

[3] M. Huhtinen, P. Lecomte, D. Luckey, F. Nessi-Tedaldi, F. Pauss, Nucl. Instrum. Meth. A545 (2005) 63-87.

[4] V. A. Batarin et al., Nucl. Instrum. Meth. A530 (2004) 286-292.

[5] E. Auffray et al. CMS-NOTE-1998-069, CERN, Geneva (Switzerland, 1998).

[6] F. Nessi-Tedaldi, Crystals for high-energy calorimeters in extreme environments, To be published in Proc. 9th ICATPP Conf. on Astroparticle, Particle, Space Physics, Detectors and Medical Physics Applications, Como (Italy, 2005), Preprint ETHZ-IPP-PR-2005-03 (2005).

[7] M. Glaser et al., Nucl. Instrum. Meth. A426 (1999) 72-77.

[8] S. Baccaro et al., $\gamma$ Irradiation facility at ENEA-Casaccia Centre (Roma), ENEA report RT/2005/28/FIS, ISSN/0393-3016, ENEA Casaccia, Rome (Italy, 2005).

[9] R. Y. Zhu, Nucl. Instrum. Meth. A413 (1998) 297-311.

[10] H. F. Chen, K. Deiters, H. Hofer, P. Lecomte, F. Nessi-Tedaldi, Nucl. Instrum. Meth. A414 (1998) 149-155. 
[11] The CMS Collaboration, The ECAL Technical Design Report, CERN/LHCC 97-33, CMS TDR 4, CERN, Geneva (Switzerland, 1997).

[12] Tyvek ${ }^{\mathrm{TM}_{\text {is }}}$ a product of DuPont.

[13] Hytec Electronics Ldt, Reading, Berkshire (England).

[14] I. Dafinei, E. Auffray, P. Lecoq and M. Schneegans, Mat. Res. Soc. Symp. Proc. 348 (1994) 99-104. 